\title{
Exploring by doing: How young chimpanzees discover surfaces through actions with objects
}

\author{
Hideko Takeshita $^{\mathrm{a}, *}$, Dorothy Fragaszy ${ }^{\mathrm{b}}$, Yuu Mizuno $^{\mathrm{c}}$, \\ Tetsuro Matsuzawa ${ }^{\mathrm{c}}$, Masaki Tomonaga ${ }^{\mathrm{c}}$, Masayuki Tanaka ${ }^{\mathrm{c}}$ \\ ${ }^{a}$ School of Human Cultures, The University of Shiga Prefecture, Hassakacho 2500, \\ Hikone, Shiga 522-8533, Japan \\ ${ }^{\mathrm{b}}$ University of Georgia, USA \\ ${ }^{\mathrm{c}}$ Primate Research Institute, Kyoto University, Japan
}

\begin{abstract}
In human infants, perception-action routines that combine objects and surfaces in playful exploration presage using objects as tools. Banging a surface with a held object is a canonical example of this phenomenon. This longitudinal study revealed when and how exploratory activity with an object emerges in chimpanzees. We studied the development of exploratory actions with objects and surfaces in three infant chimpanzees between 13 and 21 months. The infants predominantly manipulated the cube or the surface separately at 13 and 15 months. By 21 months, all infants frequently combined the objects and surfaces, although they performed less differentiated actions with them than did an adult chimpanzee. Most of the infants' combinatorial actions were relatively gentle, involving placing and releasing rather than banging as seen in humans. Frequency of combination was affected with properties of surfaces rather than of objects.
\end{abstract}

(C) 2005 Elsevier Inc. All rights reserved.

Keywords: Perception-action routine; Chimpanzee; Combinatory manipulation; Tool use

\section{Introduction}

Animals explore to learn about their environments, which permits them to regulate their activity in biologically relevant ways (Gibson, 1966; Reed, 1996). Human infants employ characteristic actions

\footnotetext{
* Corresponding author. Tel.: +81 74928 8444; fax: +81 749288559.

E-mail address: hideko@shc.usp.ac.jp (H. Takeshita).
} 
(perception-action routines) such as sucking, kicking, or banging, to explore what they can do with their bodies and how they can interact with the social and physical environment (Rochat, 2001; Thelen \& Smith, 1994). Infants begin to include objects in exploratory perception-action routines at around 6 months of age, e.g., banging an object on a table. Activities combining objects and surfaces become increasingly more diverse and complex with development. Perception-action routines that combine objects and surfaces allow infants to discover how to relate objects and surfaces through actions. Producing varied actions combining objects and surfaces allows an individual to learn about the potential for acting with objects by experiencing what happens. Adult humans can do this very efficiently with objects that can be held in one hand and manipulated at will - for example, paper clips, fork, hair brush, hammer, etc.

How do infants learn to organize actions relating objects and surfaces? Where does the process start? According to perception-action theory (Lockman, 2000): (1) developmentally, perception-action routines involving direct action on objects will appear earlier than routines that combine objects, or objects and surfaces, because these latter routines demand more attentional and perceptual resources; (2) developmentally, perception-action routines are initially rather general, but become differentiated so that actions with particular kinds of objects and surfaces take advantage of particular properties, and so that positioning of objects and surfaces with respect to one another becomes more specific and more precise; (3) young or novice individuals will develop increasingly adult forms of combining objects and surfaces when they (a) use species-typical perception-action routines with the relevant objects and surfaces, (b) differentiate actions with various surfaces and objects, and (c) coordinate or orient objects and surfaces to one another in more differentiated fashion.

The developmental process leading from simple perception-action routines combining objects and surfaces to skilled instrumental behavior is likely shared among species that routinely act upon surfaces and otherwise make use of combinatorial actions in their daily lives. Chimpanzees are among these species, as are humans. They are well known as capable tool users both in the wild and in captivity. They show similar tool-use behaviors using similar actions comprised with similar morphological bodyparts to those in humans. Although several studies describing acquisition or development of tool use in chimpanzees have been published (Bard, Fragaszy, \& Visalberghi, 1995; Celli, Tomonaga, Udono, Teramoto, \& Nagano, 2001; Inoue-Nakamura \& Matsuzawa, 1997; Lonsdorf, 2001; McGrew, 1977; Menzel, Davenport, \& Rogers, 1970; Sumita, Kitahara-Frisch, \& Norikoshi, 1985; Takeshita \& van Hooff, 2001; Tonooka, Tomonaga, \& Matsuzawa, 1997) and combinatory manipulation has been considered to be a precursor of tool use (Fragaszy \& Adams-Curtis, 1991; Takeshita, 2001; Torigoe, 1985), we lack empirical studies focusing with chimpanzees on the development of exploratory perception-action routines combining objects and surfaces, and their relationship to the development of tool use (Hayashi \& Matsuzawa, 2003).

We report in this paper when and how infant chimpanzees come to combine objects with surfaces and how combinatory actions develop prior to development of tool use. We were interested in evaluating the specific predictions generated from perception-action theory, and also in describing the general nature of young chimpanzees' explorations of objects and surfaces. perception-action theory predicts that (1) young chimpanzees, like human infants, will first use direct actions with one object or surface at a time; perception-action routines that combine objects and surfaces will appear later, (2) initially, young chimpanzees' actions with objects will be non-specific; that is, the actor will not take advantage of a special property of an object or surface. For this purpose, we examined how infants begin to establish relations between objects and surfaces when they manipulate objects, and whether they take into account 
the relation between the physical composition of the object and the property of the surface with which the object makes contact, i.e., its rigidity, elasticity, solidity, or continuity.

\section{Method}

\subsection{Participants}

Three infant chimpanzees, one male (Ayumu) and two females (Cleo and Pal) participated in the study (see Table 1). They were born in the Primate Research Institute, Kyoto University, Japan, and have been raised by their biological mothers living in a community of 11 members. The mothers have had abundant experience with various types of cognitive experiments and maintain a close relationship to human experimenters. Based on these relationships, various types of experiments investigating the infants' development have been conducted with the human experimenter and the chimpanzee motherinfant pair together in a face-to-face setting since the infant's birth. In our experiment, mothers and infants voluntarily entered an indoor room and interacted with the human experimenter during an hourly session once or twice daily. Our protocol occupied 15-20 min of an hourly session. Our testing was enabled by the mother chimpanzee's cooperation in retrieving objects taken by her infant and returning them to the experimenter, and occasionally by bringing the infant back to the testing site from the far reaches of the room.

\subsection{Material, design and procedure}

We presented each infant with a cube $(2.5 \mathrm{~cm}$ on each side) and an experimental surface $(42.8 \mathrm{~cm} \times 38.0 \mathrm{~cm} \times 5.0 \mathrm{~cm})$ covered with a specific material. Two kinds of cubes, i.e., wood (hard), or sponge (soft), and four kinds of surface materials, i.e., wood (hard), sponge (soft), water to a depth of $3 \mathrm{~cm}$ (liquid), or nylon netting with openings of $2 \mathrm{~cm}$ (discontinuous) were used (Fig. 1). We presented uniform surfaces (all one material) and non-uniform surfaces (adjacent equal-sized sections of wood and sponge, wood and water, or wood and netting). Cubes were presented one at a time with each of the four uniform surfaces in random order for a total of eight trials. Then, the two cubes were presented in sequence with the three non-uniform surfaces in random order for a total of six trials.

For each trial, the surface was placed on the floor and the cube was placed nearby. The infant was free to act with either the cube or the surface, or neither, for 1 min. Thus, infants had 14 1-min trials in sequence on each test session. During each session, the human experimenter exhibited one action five times with the wood cube on one uniform surface, and on that same surface when it first appeared in

Table 1

Participants

\begin{tabular}{lllll}
\hline Name & Name of mother & Date of birth & Test periods & Ages tested (months) \\
\hline Ayumu & $\mathrm{Ai}$ & $24 / 04 / 2000$ & $14 / 09 / 2001-29 / 01 / 2002$ & 17,21 \\
Cleo & Cloe & $09 / 06 / 2000$ & $17 / 09 / 2001-04 / 04 / 2002$ & $15,17,21$ \\
Pal & Pan & $09 / 08 / 2000$ & $13 / 09 / 2001-06 / 06 / 2002$ & $13,17,21$ \\
\hline
\end{tabular}




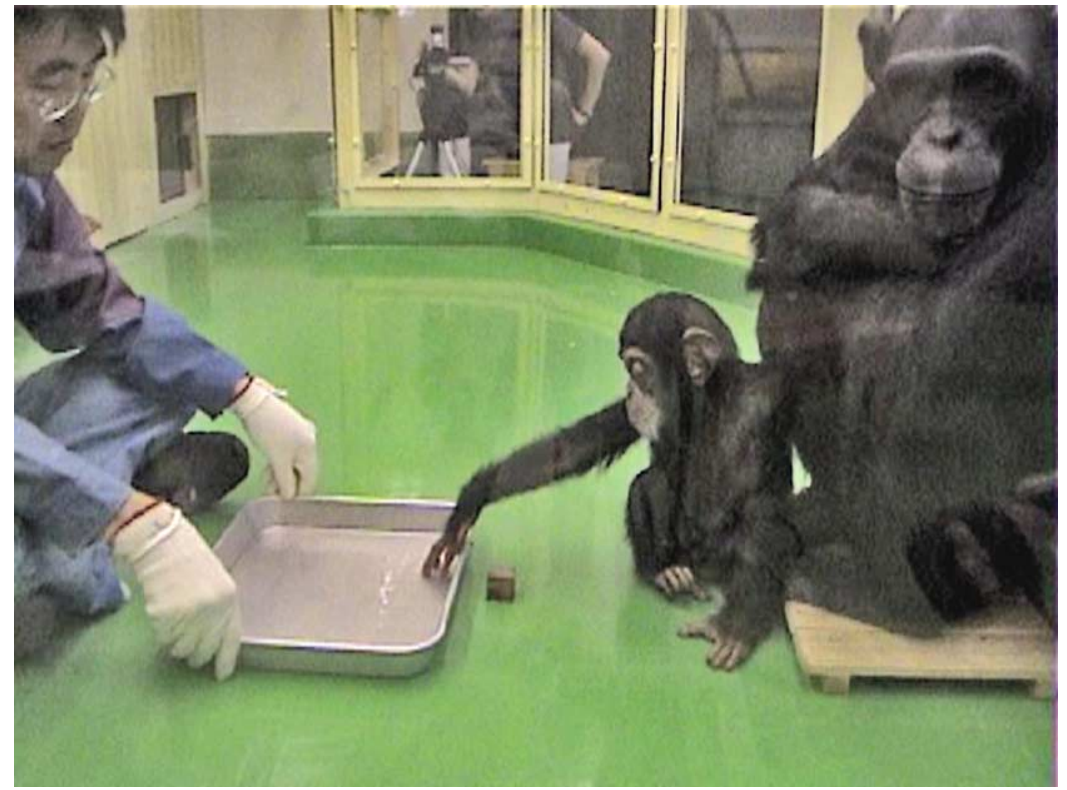

Fig. 1. A still image showing subject Pal touching a pan of water with her mother nearby at 13 months. A wood cube was presented in front of water surface: a pan with water to a depth of $3 \mathrm{~cm}$.

the non-uniform surface condition. The action demonstrated was unique for each infant. Ayumu saw the experimenter hit a cube on the wood surface. Cleo saw the experimenter press a cube into the sponge with the back of his fingers. Pal saw the experimenter stroke the water with the cube.

We completed two to three test sessions for each condition in this fashion over the course of about 10 days when the infants were 13-15 months, 17 months, and 21 months old. In the present paper we utilize the data obtained in the uniform condition. Ayumu was 17 months old when testing began, and so he has no scores for 13-15 months. Pal received two sessions at 17 months. All other replications incorporated three test sessions per infant. A total of 23 test sessions were completed, 6 with Ayumu, 9 with Cleo, and 8 with Pal. We videotaped all the trials. In the first testing period, the mothers were allowed to interact with the test objects and surfaces for varying lengths of time after the infants' 1-min sessions were completed. The mothers' activities with the cubes and surfaces were also videotaped.

\subsection{Transcription and scoring}

We scored from video each action the baby made with the cube or with the experimental surface for $1 \mathrm{~min}$ after the presentation of the materials. An action was defined as one cycle of movement. For example, when pushing the cube with one finger, each push was one action, and when rubbing the surface with the cube, each cycle of forward/back was one action. Actions were grouped into five categories: (1) manipulation of cube only; (2) manipulation of surface only; (3) manipulation of cube on experimental surface; (4) combinatory manipulation of cube with experimental surface in active fashion (hit, slide, rub, etc.); and (5) combinatory manipulation of cube with surface other than the experimental surface. We scored many varieties of actions within each category but this paper focuses on these five categories. We 
also scored the activity of one mother (Pan, Pal's mother), that was the most active performer among the three mothers and showed strong interest in manipulating objects, at the first test session, to provide a quantitative comparison to the infants' activity. Data were collated by trial.

\subsection{Analysis}

Because Pal's performance was extremely inactive in the first session of the first test period, her data in this session were regarded as an outlier and excluded from further quantitative analysis. Frequency data were log-transformed to achieve normal distribution and equivalent variance, as confirmed with the Shapiro-Wilk test and the Levene test respectively. Following transformation, we analyzed variations in the frequency of actions with the cube (category 1 and categories 3-5) using a repeated measures design. The model used session as the unit of analysis and specified the main factors cube material (within-subjects variable: 1 d.f.), age groups (between-subjects variable: 2 d.f.), subjects (between-subjects variable: 2 d.f.), and the interaction of three combinations of two factors $(2$ d.f. for subject $\times$ cube, 2 d.f. for age $\times$ cube material, and 3 d.f. for subject $\times$ age group) and one combination of all three factors ( 3 d.f.), with the remaining degrees of freedom (14 d.f.) used as the error term. We used Bonferroni post-hoc tests to explore the sources of significant main effects. We analyzed variation of frequency of contact with the surface (category 2) across subjects and age groups using the non-parametric Steel-Dwass test. A doubly multivariate repeated measures design was used to compare the distribution of actions across cube and surface conditions. The model used subject as the unit of analysis and specified the main factors cube (within-subjects variable: 1 d.f.) and surface (within-subjects variable: 3 d.f.), and the interaction the two main factors (3 d.f.) with the remaining degrees of freedom $(6,18$ and 18 d.f., respectively) used as the error term. We used a repeated measures contrasts test to explore the sources of significant main effects. All statistically significant results from our parametric analyses that we report here had a Cohen's $f$ of at least 0.25 , within the range of conventionally accepted effect sizes in the psychological literature. Values for Cohen's $f$ ranged from 0.29 to 0.76 . We used chi-square tests to evaluate the distribution of combinatorial and non-combinatorial activities across the three infants and the one mother whose activity we scored, and also among three test periods.

\section{Results}

\subsection{Comparing mothers and infants}

The infant chimpanzees exhibited strong interest in the materials, but they explored the materials in strikingly different ways from their mothers. Mothers used the cubes to explore the surfaces, or they used them in ways that were appropriate for each kind of cube and surface. For example, mothers rubbed the sponge surface with a sponge cube; soaked a sponge cube in water and then wiped the floor or themselves; and inserted the wood cube through the netting with one hand while holding the netting taut with the fingers of the other hand. Infants rarely combined the cubes with surfaces but rather explored cubes and surfaces directly. For example, they mouthed the cubes and rubbed or hit the surfaces with their hands.

Fig. 2 presents the manipulatory behaviors of the three infants and one mother, Pan during the first test period, when the infants were between 13 and 17 months old. The distribution of non-combinatory 


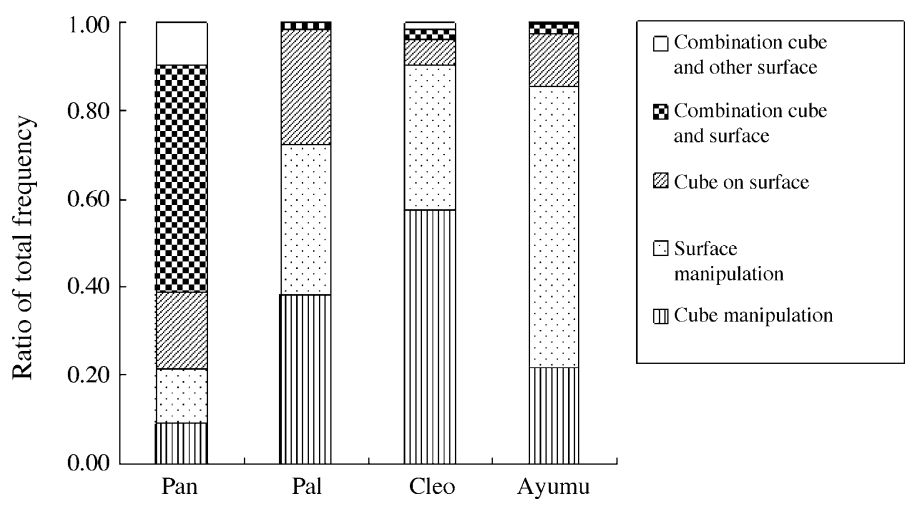

Fig. 2. Proportional distribution of five categories of manipulation in an adult female (Pan) and three infants at 13 (Pal), 15 (Cleo), and 17 (Ayumu) months of age, respectively. (1) Cube manipulation: manipulation of cube only; (2) surface manipulation: manipulation of surface only; (3) cube on surface: manipulation of cube on experimental surface; (4) combination cube and surface: combinatory manipulation of cube with experimental surface in active fashion (hit, slide, rub, etc.); and (5) combination cube and other surface: combinatory manipulation of cube with surface other than the experimental surface.

(categories 1-3) and combinatory manipulation (categories 4 and 5) differed significantly among the four individuals $\left(\chi^{2}=437.87\right.$, d.f. $\left.=3, p<0.01\right)$. More than half of the mother's actions were categories 4 and 5 , whereas all three infants combined objects and surfaces rarely.

\subsection{Developmental changes: comparing infants across ages}

Infants manipulated the cubes frequently throughout the testing periods, from an average of 5.0 times per minute at 13-15 months to an average of 9.0 times per minute at 21 months. Fig. 3 shows mean frequency of contact with each cube per minute for each age group. The main effect of subject was significant, $F(2,14)=7.079 ; p=0.008$. The Bonferroni test revealed that Pal showed more cube manipulation

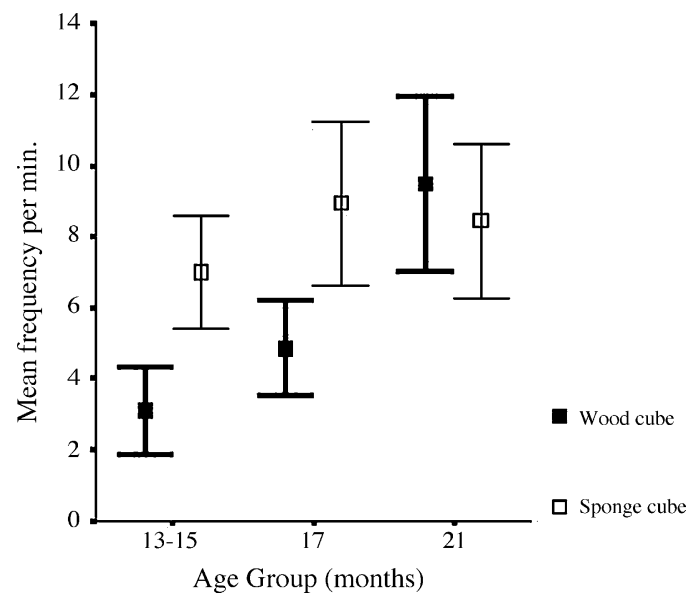

Fig. 3. Mean frequency of manipulation of wood or sponge cube per subject per one-minute trial. $N$ per age varied from 5 (13-15 months), to 8 (17 months) or 9 (21 months). Bars indicate mean + SEM and mean - SEM. 


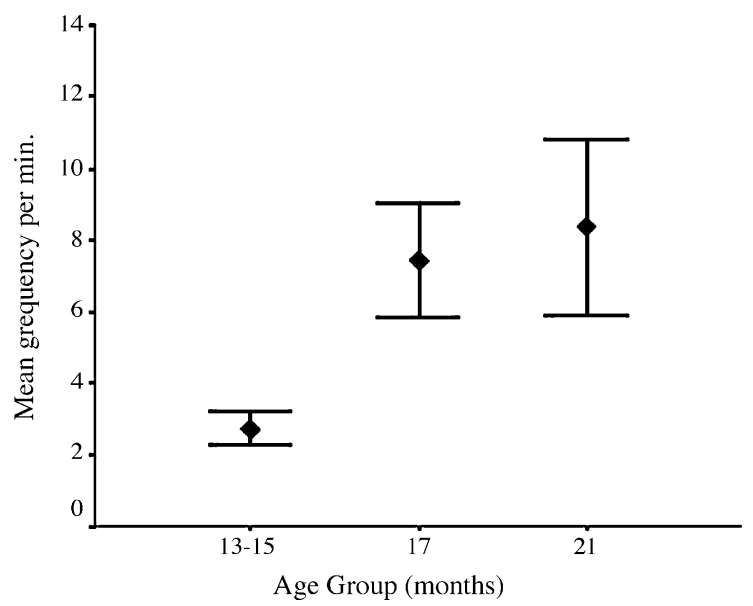

Fig. 4. Mean frequency of contact with the experimental surfaces per subject per one-minute trial. Sample sizes are as that in Fig.3. Bars indicate mean + SEM and mean - SEM.

than Cleo ( $p=0.002)$. The main effect of Cube was also significant, $F(1,14)=10.354, p=0.008$, and the main effect of Age was not. Because cube $\times$ age interaction was significant, $F(2,14)=5.652 ; p=.022$, we looked at simple main effects of Cube at each age group. The infants manipulated the Wood cube significantly less frequently than the Sponge cube at $13-15$ months and 17 months, $F(1,28)=10.801$, $p<0.005$ and $F(1,28)=10.205, p<0.005$, respectively.

Fig. 4 provides mean frequency of contact with the experimental surface per minute for each age group. The infants contacted surfaces nearly as frequently as they manipulated cubes throughout the experiment. However, this variable did not differ significantly across subjects or age groups (Steel-Dwass test, $T=-1.9926$ to $1.9926, p>.05$ ).

Through 17 months, infants primarily explored cubes directly and surfaces directly, although they placed the cube on the surface in $2-7 \%$ of actions at the first two test periods. Infants at $13-17$ months

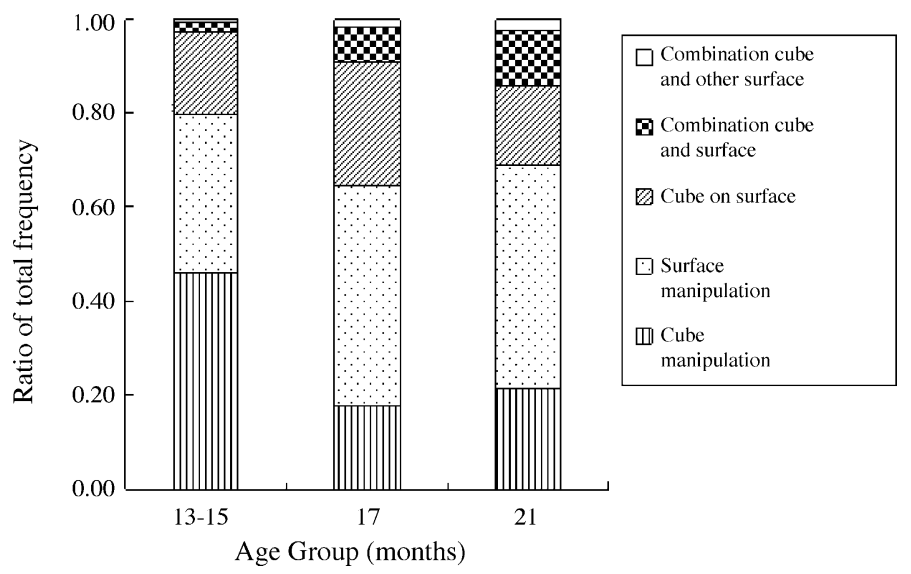

Fig. 5. Proportional distribution of five categories of manipulation by infants at three different ages. Categories of manipulation are as that in Fig. 2. 
often mouthed both kinds of cubes and only rarely combined a cube with any surface other than by placing it on the surface. At 21 months, however, combinatorial activity became more common, constituting about $12 \%$ of all activity (see Fig. 5). The distribution of activity across combinatory manipulation (categories 4 and 5) and non-combinatory manipulation (categories $1-3)$, differed significantly with age $\left(\chi^{2}=39.88\right.$, d.f. $=2, p<0.01$ ). For two babies, Ayumu and Pal, combinatory manipulation increased between 17 and 21 months. For the third baby, Cleo, the increase occurred between 15 and 17 months, and activity was stable between 17 and 21 months.

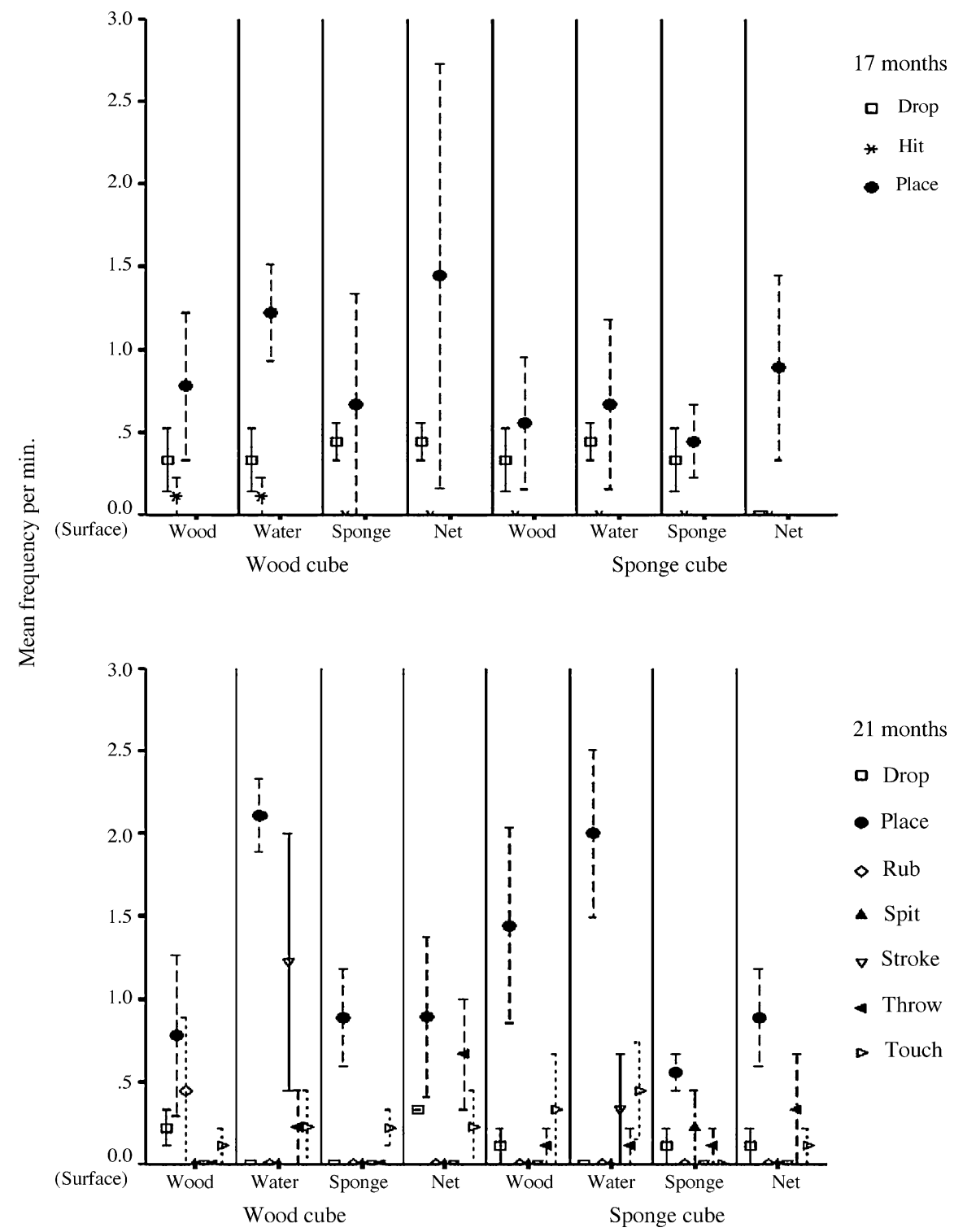

Fig. 6. Mean frequency of various actions combining the wood or sponge cube and experimental surfaces per subject per one-minute trial. Above: 17 months. Below: 21 months. Bars indicate mean + SEM and mean - SEM. 


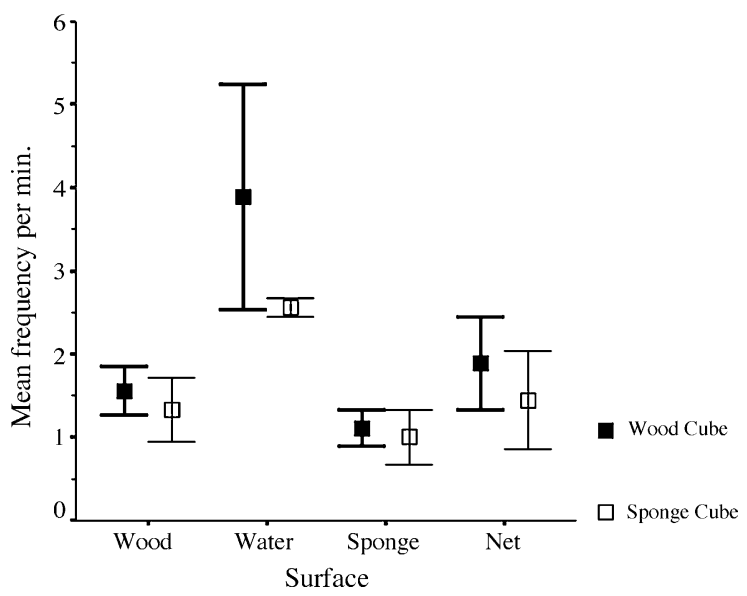

Fig. 7. Mean frequency of actions combining a cube with the experimental surfaces per subject per one-minute trial at 21 months. Bars indicate mean + SEM and mean - SEM.

\subsection{Comparing combinatory manipulation with different objects and surfaces}

Fig. 6 shows the action repertoire observed in combinatory manipulation at 17 and 21 months in all the infants. At 17 months, combinatory manipulation emerged with the actions leading to single, momentary combination of the cube and surface such as 'drop', 'hit', and 'place'. At 21 months, however, 'place' remained frequent, but other actions such as 'rub', 'stroke', and 'touch', which lead to more continuous of combination of the cube and the surface, occurred more frequently than at 17 months. Dominant combinatory manipulation at 17 months involved a single action to release an object on the surface. At 21 months, however, objects were frequently brought repetitively into contact with the surface in cycles of rubbing, sweeping, or banging.

We looked at whether the total frequency of combinatory manipulation per subject at 21 months differed depending on experimental conditions (see Fig. 7). A repeated measure design ANOVA for dual multi-variables revealed a significant main effect for Surface, $F(3,18)=4.973 ; p=0.046$, but other main effects and any interactions were not significant. A repeated measures contrasts test revealed significant difference between water surface and wood surface, $F(1,6)=32.161 ; p=0.030$. Slight differences between water surface and sponge surface, $F(1,6)=11.303 ; p=0.078$, and between water surface and net surface, $F(1,6)=11.250 ; p=0.079$, were seen. Infants contacted the water with a cube more often than any other surface (mean frequency per minute per subject $=3.78$, water, versus $1.11-2.11$, other surfaces).

\subsection{Effect of demonstrations}

Only one infant routinely performed the action demonstrated by the human experimenter. Recall that Ayumu saw the experimenter bang the wood cube on the wood surface. Ayumu hit the wood surface with a wood cube only once at 17 months and he did not perform this action at 21 months. Cleo observed the wood cube being pressed into the sponge surface, but she never pressed the sponge with either cube during any trial. Pal saw the experimenter stroke the water with the wood cube. She stroked the water with wood and sponge cubes frequently at 21 months. Pal did this significantly more frequently than Ayumu 
at the same age (11 times versus 3 times; $\chi^{2}=4.57$, d.f. $\left.=1, p<0.05\right)$. Cleo showed this only once during the three test periods at 17 months. Pal's mother spent much time in early test sessions (when Pal was 13 months old) placing and moving objects in the water when Pal's trials were finished. Her performance was restrained in later sessions.

\section{Discussion}

Previous studies on development of object manipulation in chimpanzees reported delay of emergence of combinatory manipulation when compared with human infants (Poti \& Spinozzi, 1994; Takeshita, 2001; Vauclair \& Bard, 1983). Human infants start banging against a table with an object, e.g., a rattle, at around 7 months, and placing an object on a table, into a cup, or on another object, at around 10 months. We predicted that young chimpanzees would first use direct actions with one object or surface at a time and perception-action routines to combine objects and surfaces would appear later, as in humans. This was supported. Infant chimpanzees at the PRI achieved a shift in how they acted with objects at around 17-21 months. They shifted from direct action on an object or surface to a variety of actions combining objects and surfaces. They also showed some differentiation of actions according to surfaces by 21 months. The development of variety and complexity in manipulation of a single object or surface might be a prerequisite to emergence of combinatory manipulation in chimpanzee infants, as it is in humans (Takeshita, 1999).

Aside from confirming the delay in appearance of combinatorial manipulation in young chimpanzees in comparison to humans, our study illustrates the dramatic differences between the two species in the typical form of early combinatorial actions. Human infants begin to bang objects against surfaces before their first birthday, and this action remains a ubiquitous element in the human manipulative repertoire thereafter (Lockman, 2000). In contrast, our young chimpanzee subjects did not bang cubes against surfaces; most of their combinatorial actions were far gentler. These observations, together with those of Inoue-Nakamura and Matsuzawa (1997) describing the relative rarity of striking actions in the manipulative repertoire of young wild chimpanzees, indicate that young chimpanzees explore object-surface combinations in a fundamentally different way than do young humans. The differences in the characteristic forms of combining objects and surfaces presage differences in the forms of tool use discovered at an early age and widespread in each species: hammering in humans, versus insertion in chimpanzees (as in using sticks or blades of grass to fish for ants or termites, or leaves to sponge up water, common forms of tool use in wild and captive chimpanzees alike; McGrew, 1992; Takeshita and van Hooff, 2001; Lonsdorf, 2001).

Based on the present results, we identify two phases in the early development of combinatory manipulation in chimpanzees. In the first phase the infants explore contacting an object with a surface. In the second phase infants explore how the contact can be regulated through their own actions. We predict that these phases developmentally precede and overlap with the emergence of tool use, as formulated by Lockman (2000). Lockman (2000) argued that the development of tool use may be formulated as a two-fold problem of (1) detecting and (2) relating affordances. From this perspective, tool use emerges "from infants' early and continual efforts to detect and act on the affordances of the world around them" (Lockman, 2000, p. 140). Detecting affordances refers to recognizing that an object can be combined with, or related to, another object or surface to achieve a goal. Relating affordances refers to producing the appropriate relation between objects and surfaces to achieve a goal. 
Chimpanzee infants in the present study started to combine an object with surfaces through a few actions that seemed intended to achieve simple contact of object to surface. They discovered that an object can be 'on' various surfaces through the actions of dropping, or placing during the first half of the second year. At 21 months, their combinatory actions took on the characteristics of active exploration of forms and consequences of the contact. They still most often related an object with various surfaces through the same action, placing (an indirect form of touching). However, they explored the water surface with an object differently from the other surfaces, by stroking, which led to licking water off the cube. In this sense, they began to use the cube as a tool. Exploring the water by stroking allowed them to discover the unusual properties of water compared to other surfaces. However, they did not establish differentiated actions relating specific objects and surfaces. For example, they did not place the sponge cube into the water more often than the wooden cube, although a sponge cube absorbs more water. This result suggests that infants initially explore surfaces using the same actions with any object, without differentiating actions according to the properties of the held object.

Infants began to differentiate surfaces through action, and direct actions preferentially at certain surfaces, at 21 months. At this age, water was the most attractive surface to generate combinatory manipulation. This finding suggests that chimpanzee infants recognize properties of surfaces through actions with different objects before knowing specific properties of objects. They may learn the latter through accumulated experience with combinatory manipulation with different objects.Three other studies with the infant subjects of this study provide complementary findings that help us to interpret our results. First, Hayashi and Matsuzawa (2003) studied combinatory manipulation in the same infants in a different setting (play with a posting box). Each infant first put a slender stick into a circular hole in the posting box, a form of combinatory manipulation, at 8-11 months. However, this action remained rare in all the infants until about 18 months. The frequency and variety of combinatory manipulation with the posting box and other objects used in Hayashi and Matsuzawa's study increased from around 18 months onwards, as it did in ours. Second, the same three infants succeeded in placing sticks and other long, thin objects into a narrow opening to "dip" for honey from the age of 20 months (Hirata \& Celli, 2002). In Hirata and Celli's study, the infants encountered a wide variety of objects in the company of adults (including their mothers) actively dipping for honey by probing an object through a hole in the wall, and the infants had access to identical holes and objects concurrently with the adults. The supportive social setting and opportunities for concurrent action no doubt contributed to the infants' persistent exploration of objects and surfaces and their discovery at a young age of an effective action linking stick and hole to the retrieval of honey. Finally, Tomonaga, Mizuno and Hayashi (2003) found that Cleo, one of the subjects of the present study, at age 17 months showed more combinatory manipulation when she confronted a tray with water than when she confronted a tray without water, and she used a paper towel to drink water more often than she used other objects for this purpose. With the water tray and a paper towel, species-common combinatory actions such as placing produce effective outcomes, and this likely promotes the development of actions taking advantage of interesting or useful affordances. All three of these studies illustrate how perception-action routines can promote the discovery of how to combine objects and surfaces in goal-directed action.

Does others' behavior influence exploratory actions by infant chimpanzees? The answer is no, at least not in the space of the 1-min trial period used in this study. Only one infant reproduced the model behavior demonstrated by a human experimenter, stroking the cube through the pan of water, and she did so at 21 months, but not before. The behavior was also performed by her mother when she had access to the water, whereas the other modeled actions were not performed by the mother chimpanzees. Stroking the cube 
through water was also seen in the other infants at this age. Thus, overall, we found no strong evidence that a modeled action influenced the infants' immediately subsequent activity. However, the contributions of social partners to support specific perception-action routines in chimpanzee infants under two years of age deserve further inquiry. We predict that species-typical perception-action routines channel how young individuals act on the environment, and that species-common actions relating objects and surfaces or other objects underlie the development of tool use in chimpanzees and humans, and indeed, in all the many species exhibiting tool use. Social partners most likely exert influence on the development of tool use through their modulation of young individuals' spontaneous exploratory activities.

\section{Acknowledgements}

We thank staff of the Section of Language and Intelligence, Primate Research Institute, Kyoto University, Japan, for their helpful assistance during this project. The study was financially supported by grants from the Ministry of Education, Science, Sports, and Culture, Japan (grant \#12002009 to T. Matsuzawa and \#13015143 to H. Takeshita), grants for cooperative studies at the PRI from Kyoto University, and by Research Fellowship from Japan Society for the Promotion of Science and Heiwa Nakajima Foundation to D.M. Fragaszy.

\section{References}

Bard, K. A., Fragaszy, D., \& Visalberghi, E. (1995). Acquisition and comprehension of a tool-using behavior by young chimpanzees (Pan troglodytes): effects of age and modeling. International Journal of Comparative Psychology, 8, 47-68.

Celli, M. L., Tomonaga, M., Udono, T., Teramoto, M., \& Nagano, K. (2001). Learning processes in the acquisition of a tool using task by captive chimpanzees. Psychologia, 44, 70-81.

Fragaszy, D. M., \& Adams-Curtis, L. E. (1991). Generative aspects of manipulation in tufted capuchin monkeys (Cebus apella). Journal of Comparative Psychology, 105, 387-397.

Gibson, J. J. (1966). The senses considered as perceptual systems. Boston: Houghton Mifflin.

Hayashi, M., \& Matsuzawa, T. (2003). Development of orienting manipulation in infant chimpanzees. Animal Cognition, 6, $225-233$.

Hirata, S., \& Celli, M. L. (2002). Learning of tool use by infant chimpanzees: The role of mothers on the acquisition of knowledge. Caring for primates. In Proceedings of the Abstracts of The XIXth Congress. The International Primatological Society (pp. 55-56).

Inoue-Nakamura, N., \& Matsuzawa, T. (1997). Development of stone tool use by wild chimpanzees (Pan troglodytes). Journal of Comparative Psychology, 111, 159-173.

Lockman, J. J. (2000). A perception-action perspective on tool use development. Child Development, 71, $137-144$.

Lonsdorf, E. V. (2001). Development and acquisition of termite-fishing skills in the Gombe chimpanzees. In The apes: Challenges for 21 st century. Conference Proceedings (pp. 327-330), Brookfield: Brookfield Zoo.

McGrew, W. C. (1977). Socialization and object manipulation of wild chimpanzees. In S. Chevalier-Skolnikoff \& F. E. Poirier (Eds.), Primate bio-social development: Biological, social, and ecological determinants (pp. 261-288). New York: Garland Publishing.

McGrew, W. C. (1992). Chimpanzee material culture: Implications for human evolution. New York: Cambridge University Press.

Menzel, E. W., Jr., Davenport, R. K., \& Rogers, C. M. (1970). The development of tool using in wild-born and restriction-reared chimpanzees. Folia Primatologica, 12, 273-283.

Poti, P., \& Spinozzi, G. (1994). Early sensorimotor development in chimpanzees (Pan troglodytes). Journal of Comparative Psychology, 108, 93-103.

Reed, E. S. (1996). Encountering the world: Toward an ecological psychology. New York: Oxford University Press. 
Rochat, P. (2001). The infants' world. Cambridge, MA: Harvard University Press.

Sumita, K., Kitahara-Frisch, J., \& Norikoshi, K. (1985). The acquisition of stone-tool use in captive chimpanzees. Primates, 26, $168-181$.

Takeshita, H. (1999). Early development of human mind and language: Comparative studies of behavioral development in primates. Tokyo: University of Tokyo Press [in Japanese].

Takeshita, H. (2001). Development of combinatory manipulation in chimpanzee infants (Pan troglodytes). Animal Cognition, 4, $335-345$.

Takeshita, H., \& van Hooff, J. A. R. A. M. (2001). Tool use by chimpanzees (Pan troglodytes) of the Arnhem Zoo community. In T. Matsuzawa (Ed.), Primate origins of human cognition and behavior (pp. 519-536). Tokyo: Springer.

Thelen, E., \& Smith, L. B. (1994). A dynamic systems approach to the development of cognition and action. Cambridge, MA: MIT Press.

Tomonaga, M., Mizuno, Y., \& Hayashi, M. (2003). Emergence of drinking water behavior using a tool in an infant chimpanzee. In M. Tomonaga, M. Tanaka, \& T. Matsuzawa (Eds.), Cognitive and behavioral development in chimpanzees: A comparative approach (pp. 146-152). Kyoto: Kyoto University Academic Press [in Japanese].

Tonooka, R., Tomonaga, M., \& Matsuzawa, T. (1997). Acquisition and transmission of tool making and use for drinking juice in a group of captive chimpanzees (Pan troglodytes). Japanese Psychological Research, 39, 253-265.

Torigoe, T. (1985). Comparison of object manipulation among 74 species of non-human primates. Primates, 26, 182-194.

Vauclair, J., \& Bard, K. A. (1983). Development of manipulations with objects in ape and human infants. Journal of Human Evolution, 12, 631-645. 